\title{
INTEGRACIÓN ECONÓMICA Y POLITICAS DE MIGRACIÓN: LOS DESAFÍOS PARA MÉXICO YESTADOS UNIDOS
}

\author{
ALEXANDRA DÉLANO
}

Traducción del inglés

Luis Rodolfo Morán

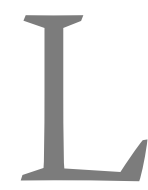

a relación bilateral entre México y Estados Unidos sufrió un importante cambio a raíz de la aprobación del Tratado de Libre Comercio de América del Norte (TLCAN) en 1994. ${ }^{1}$ La liberalización de la economía mexicana desde la década de los ochenta ya había tenido como consecuencia una integración significativa de los mercados de esas naciones, pero la firma del TLCAN indicaba que los gobiernos estaban dispuestos a establecer un acuerdo formal y extenso de cooperación comercial y financiera. Anteriormente, la asimetría de poder económico y político entre ambos países había incidido en el hecho de que Estados Unidos podía lograr sus metas en todas las áreas sin tener que asumir los costos de establecer compromisos formales con México. A su vez, México carecía del suficiente poder de negociación para influir en las políticas estadounidenses e inclinarlas hacia sus intereses. No obstante, en el caso del TLCAN, ambos países estuvieron de acuerdo en que la mejor manera de proteger sus intereses, a la luz de los cambios y las nuevas tendencias en el ámbito internacional, era a través de la cooperación económica.

Aun cuando persiste la asimetría de poder —dado que la dependencia de la economía mexicana respecto a Estados Unidos es mayor de lo que éste depende del mercado mexicano-, la cooperación iniciada en materia de comercio y finanzas ha ejercido una importante influencia en la manera en que ambos países se perciben entre sí y en las políticas internas e internacionales que ponen en práctica. Muchos analistas coinciden en que el TLCAN no es sólo un acuerdo comercial: sus implicaciones son de amplio espectro y sus consecuencias afectan a los renglones político, social y cultural de ambos países (Alba, 1998; Massey et al., 2002). A medida que en los diez años transcurridos se ha desarrollado el proceso de cooperación económica, se amplían los límites

\footnotetext{
${ }^{1}$ En este análisis me centro sólo en la relación entre México y Estados Unidos, por lo que no se menciona a Canadá, el tercer socio del TLCAN.
} 
de coordinación de políticas hacia otras áreas, las que en algunos casos incluyen temas históricamente controvertidos, como el manejo de la migración laboral documentada e indocumentada proveniente de México con destino a Estados Unidos.

En este ensayo analizo el grado en el cual la cooperación bilateral ha ejercido una influencia en las políticas de migración de ambos países. Argumento que la asimetría de poder entre México y Estados Unidos y la falta de consenso interno con respecto a los temas migratorios establecen límites a la cooperación formal en esta área. Aun así, creo que los actuales cambios en la relación bilateral, en especial desde la entrada en vigor del TLCAN, pueden derivar en un nuevo y mejor manejo de la situación en el futuro. En las tres secciones de este trabajo desarrollo estas ideas. Primero analizo las razones por las que el TLCAN no incluyó políticas migratorias comunes y cómo los gobiernos trataron la integración económica en relación con este tema. Por el hecho de que los flujos migratorios entre los dos países tienen causas y consecuencias económicas directas, éstas constituyen un factor relevante para lograr las metas establecidas en el TLCAN y para reforzar la integración posterior. Las razones por las que no se incluyó en el tratado una política migratoria compartida apuntan hacia ciertos factores que han obstaculizado históricamente la posibilidad de realizar un manejo bilateral del fenómeno. Con el objeto de explorar estos factores, en la segunda sección explico las circunstancias internas que determinaron las posiciones de México y Estados Unidos sobre el tema migratorio a lo largo del siglo Xx. La tercera sección se centra en los cambios que el TLCAN ha generado en el manejo y discusión del tema e incluye un análisis de la situación actual después de los ataques del 11 de septiembre de 2001. En mis comentarios finales esbozo lo que considero desafíos actuales para el manejo de la migración a corto y largo plazo.

Mi perspectiva se basa en la suposición de que los actuales flujos de la migración mexicana hacia Estados Unidos implican factores económicos, políticos y sociales vinculados a ambos países. Debe tomarse en cuenta la existencia de todas estas variables para entender en términos más amplios las circunstancias que empujan y atraen a la migración y la manera en la que son tratados en cada país. Anteriormente, uno de los mayores límites para el manejo de la migración ha sido la falta de información objetiva compartida. Ello desemboca en la creación de mitos que presentan a los migrantes como amenazas para los países receptores, en ideas sin fundamento acerca de sus efectos económicos y en políticas inadecuadas que se alejan de la realidad de la situación (Cornelius, 2000). Ya no pueden ignorarse por más tiempo las consecuencias negativas de estas tendencias para ambos países. Mi propósito es aportar elementos para aclarar la discusión con un análisis objetivo y extenso de la historia y las políticas de cada país, con la intención de enfrentar los desafíos que están implícitos en el proceso de integración entre México y Estados Unidos desde la firma del TLCAN.

\section{EL TLCAN Y LA MIGRACIÓN}

Durante las negociaciones del TLCAN, iniciadas en 1991, los presidentes George Bush (1988-1992) y Carlos Salinas de Gortari (1988-1994) acordaron restringir las discusiones a los asuntos comercial y financiero. Ambos creían que sería demasiado grande el costo político de incluir a la migración en la agenda y que podría constituirse en un obstáculo insalvable para llevar a término el TLCAN. Estuvieron de acuerdo en que los problemas causados y derivados de la migración de trabajadores mexicanos hacia 
Estados Unidos podrían resolverse indirectamente por el TLCAN. El desarrollo económico a través de la liberalización y la integración de mercados eliminaría las presiones para la emigración desde México. El crecimiento económico en México, estimulado por los flujos de inversión de capital, aportaría más trabajos y mejores salarios, eliminando con ello los incentivos para la emigración.

Esta solución se basaba en un análisis limitado de la dinámica migratoria y los diversos aspectos que deben tomarse en cuenta para generar una solución real. Implicaba que la migración sería sólo el resultado de los problemas económicos de México y suprimía el tema de la demanda permanente de mano de obra barata de parte de los patrones en Estados Unidos. Sin reconocerlo explícitamente, las estrategias de ambos gobiernos aseguraban que los flujos de migración indocumentada seguirían como en el pasado. No habría una política explícita para manejar y controlar la contratación de mexicanos en Estados Unidos, lo que implicaba que seguirían llegando a Estados Unidos bajo una condición vulnerable, sujetos a abusos y sin los beneficios de la protección laboral. Incluso de esta forma, el gobierno mexicano podía asegurar todavía el flujo de remesas y conservar una alternativa al desempleo. Para Estados Unidos, el asegurar la mano de obra de los migrantes sin contratos o acuerdos formales generaba una solución barata a su escasez de fuerza de trabajo (en especial para los empleos de bajos ingresos). Incluso cuando los sindicatos se veían afectados negativamente por la presencia de los trabajadores, su presión no fue suficiente para lograr un contrapeso al apoyo que los patrones de los trabajadores indocumentados daban a las políticas del gobierno.

Esa era la realidad de los flujos migratorios - del conocimiento de ambos gobiernos-, pero en el discurso se le disfrazaba para hacerla coincidir con los intereses políticos y económicos que estaban en juego durante las negociaciones del TLCAN. Desde el lado mexicano, Salinas seguía las tácticas que habían utilizado para evitar las tensiones diplomáticas con Estados Unidos y las críticas internas al tratar el tema de la migración. Desde los años setenta, el gobierno mexicano había recurrido al discurso retórico en el sentido de que «México quiere exportar bienes, no personas». Por un lado, esto garantizaba el apoyo económico y político de Estados Unidos para el mantenimiento de las políticas del gobierno mexicano. Por el otro, debilitaba las discusiones acerca de la carencia de políticas migratorias explícitas y exitosas por parte de México y sus intentos fallidos por defender a su población en el extranjero. Estados Unidos recurría también a esta estrategia para negociar «bienes en vez de personas». Este discurso evitaría la oposición con los grupos antiinmigrantes tradicionales en Estados Unidos e, incluso, con parte de los patrones que veían un acuerdo migratorio formal como un riesgo en su acceso a trabajadores escasamente pagados y a la evasión de controles y sanciones gubernamentales por dar trabajo a migrantes indocumentados.

A pesar de que los efectos del TLCAN en la economía mexicana no pueden ser evaluados plenamente en todas las áreas, en buena parte por el hecho de que lleva vigente apenas diez años y todavía no están disponibles los datos, es obvio que la «solución» propuesta para la migración se encuentra lejos de haberse concretado. No sólo continúan las asimetrías económicas entre ambos países, sino que también han surgido grandes problemas a medida que la competencia en algunos sectores ha sobrepasado su capacidad de ajuste. ${ }^{2}$ Tal es el caso del sector agrícola, donde se sitúa la

${ }^{2}$ Cuando se cumplan los plazos para la liberalización total de la agricultura, se pondrá a prueba la 
mayor parte de las comunidades de origen de los migrantes. Una política migratoria comprensiva ha de incluir un programa para la inversión y el desarrollo en las áreas rurales de México y en las comunidades de origen, como ya sugirieron los negociadores del TLCAN, pero incluso esa medida es insuficiente. Una solución adecuada tiene que ir más allá de las explicaciones económicas tradicionales del fenómeno (es decir, las diferencias en el salario, los razonamientos de costo-beneficio, fallas en el mercado); debe tomar en cuenta todas las características de estos flujos y explorar las razones de sus cambios históricos (Massey et al., 2002).

\section{LOS MIGRANTES MEXICANOS: QUIÉNES Y POR QUÉ}

Los datos y estudios recientes sobre México y Estados Unidos muestran que las características de los flujos migratorios provenientes de México han cambiado en las décadas recientes debido a las circunstancias demográficas, políticas, económicas y sociales. ${ }^{3}$ La población de trabajadores migrantes, compuesta en el pasado principalmente por hombres de edad adulta de las áreas rurales, incluye ahora a más mujeres y niños, hombres más jóvenes y poblaciones urbanas con niveles de escolaridad más altos. El comportamiento de los migrantes ha girado hacia periodos más largos de estancia en Estados Unidos, lo que significa que disminuye la circularidad y la temporalidad de los flujos. La cifra de trabajadores indocumentados se incrementa, lo que favorece el crecimiento de traficantes (coyotes o polleros), que cobran cuotas más altas, y aumenta la falsificación de documentos, los maltratos, la violación de los derechos humanos y el número alarmante de muertes en la frontera. La comunidad mexicana-estadounidense es ahora la minoría étnica más grande en Estados Unidos y asciende a cerca de 22 millones; 14 millones son ciudadanos estadounidenses de origen mexicano, entre ocho y ocho punto cinco millones son migrantes con documentos y tres o cuatro millones residen sin documentos en Estados Unidos (Oficina del Censo de Estados Unidos, 〈http://www.census.gov»; CONAPO, 〈http://www.conapo.gob.mx»).

En su publicación más reciente, Massey, Malone y Durand documentan el hecho de que «la inmigración puede comenzar por una diversidad de razones, pero las fuerzas que inician el movimiento internacional son bastante diferentes de aquellas que lo perpetúan» (Massey et al., 2002). Algunos ejemplos de las fuerzas, que mantienen el movimiento en el caso de la migración mexicana hacia Estados Unidos, incluyen la existencia de redes familiares y de empleo que facilitan la llegada de nuevos inmigrantes, la dependencia de las remesas en México (las que en la actualidad alcanzan 12 mil millones de dólares al año) y los crecientes vínculos sociales y económicos

habilidad del gobierno mexicano para lidiar con la competencia en este sector, lo que presagia que no habrá un resultado muy positivo. Ello podría aumentar las presiones para emigrar y generar inestabilidad económica y política en México, lo que afectaría de forma considerable a ambos países y resucitaría los debates internos y las críticas al TLCAN.

${ }^{3}$ Se pueden encontrar ejemplos de estos datos en la Oficina del Censo de Estados Unidos (http:// www.census.gov), la fuente de información sobre migración (http://www.migrationinformation. org), CONAPO (Consejo Nacional de Población, http://www.conapo.gob.mx) e INEGi (Instituto Nacional de Estadística, Geografía e Informática, http://www.inegi.gob.mx). 
entre los dos países. Tanto México como Estados Unidos han fortalecido la existencia de este flujo, mismo que se ha convertido en un factor estructural para sus economías. Las políticas migratorias (o su ausencia) han influido en las formas en que se desarrolla el fenómeno y en los costos y beneficios que implica.

\section{LAS POLÍticas MigRATORIAS DE MÉXICO Y ESTAdOS UNIDOS EN EL SIGLO XX: ASIMETRÍA DE PODER Y FACTORES INTERNOS}

A lo largo de la historia de la relación bilateral entre México y Estados Unidos, por lo general, este último ha formulado y puesto en práctica las políticas de migración ante las que México reacciona casi siempre de manera pasiva. Con excepción del Programa Bracero (1942-1964), ${ }^{4}$ la cooperación formal y explícita con el gobierno mexicano raramente se considera una opción conveniente. Estados Unidos obtiene y recluta mano de obra barata y dispone de ella según las circunstancias económicas, sin asumir los costos políticos de un acuerdo. En atención con las teorías de las relaciones internacionales, éste es un caso típico de interdependencia asimétrica (o de cooperación asimétrica) en el que la parte más débil, en este caso México, por lo general no puede proteger sus intereses o ejercer presión en contra del país más poderoso (Krasner, 1990; Keohane, 1990). Aun cuando esta asimetría constituye una condición estructural en la relación bilateral, las políticas de migración de México y Estados Unidos no pueden reducirse a esta explicación. El análisis de las raíces históricas internas de sus posiciones proporciona el marco para explicar los cambios en los años recientes, a la vez que para entender los desafíos actuales para una cooperación más amplia.

Cuando se rastrean los orígenes de los flujos de la migración mexicana hacia Estados Unidos, queda claro que las variables geográficas, económicas y demográficas juegan un papel central. Era natural que al compartir una frontera de tres mil kilómetros, que incluye un territorio que perteneció a México hasta 1848, los mexicanos cruzaran la línea. La inestabilidad económica y política de México y las condiciones de pobreza en los estados rurales, durante el Porfiriato y la Revolución de 1910, obligaron a muchos campesinos a buscar alternativas, entre ellas la migración. El hecho de que a Estados Unidos le hiciera falta mano de obra para desarrollar su territorio y consolidar la economía del suroeste, aumentó los incentivos para la emigración de mexicanos en busca de empleo en una sociedad receptiva (Corwin, 1978). La mayoría de estos trabajadores aportaron su mano de obra para la agricultura, pero a medida que aumentaron su experiencia y se desarrolló la economía de Estados Unidos, su movilidad y sus redes sociales les aseguraron el acceso a otros sectores (que en la actualidad incluyen la industria, la construcción, el trabajo doméstico y los servicios).

A pesar de que Estados Unidos puso en práctica leyes que restringían la inmigración desde otros países (principalmente asiáticos), México constituyó una excepción a la regla. La demanda de mano de obra por parte de los patrones era constante y

${ }^{4}$ Durante este programa, México y Estados Unidos lograron contratar a trabajadores mexicanos («braceros») para las labores en el sector agrícola de Estados Unidos debido a la falta de mano de obra durante la Segunda Guerra Mundial. El programa fue ratificado anualmente por ambos gobiernos hasta 1964, cuando Estados Unidos decidió cancelarlo. 
se incrementó durante las guerras mundiales debido al crecimiento en la producción y al temor a la escasez de mano de obra. La fuerza de trabajo mexicana era fácil de atraer y de retornar; era barata y los migrantes eran diligentes. No obstante, la demanda de mano de obra sobrepasó las situaciones de emergencia, convirtiéndose en un factor estructural esencial para el crecimiento económico en el suroeste. Los trabajadores estadounidenses, cuando estaban disponibles, no tenían deseos de ocupar estos trabajos duros y de escasa paga, mientras que los trabajadores mexicanos siempre eran accesibles, independientemente del salario, el tipo de trabajo o las condiciones de vida.

Aunque desde principios del siglo xx existían grupos nativistas y antiinmigrantes que ejercían presión para que el gobierno dejara fuera a los pueblos «potencialmente peligrosos», los migrantes mexicanos no se convirtieron en su blanco sino hasta la década de los veinte. Las legislaciones que se derivaron de estas presiones limitaron los flujos provenientes de Asia y Europa, lo que a su vez incrementó la migración mexicana, en especial después de la Primera Guerra Mundial. A partir de entonces, cuando se suscitaban crisis económicas o políticas, la opinión pública comenzaba a notar la presencia de la creciente población mexicana, a quien culpaban por la depresión salarial, las fallas en la organización sindical, los altos costos de la beneficencia y el desempleo. El gobierno respondía a estas presiones a través de políticas como la deportación y la repatriación (como en 1921, 1929 y 1954), legislaciones restrictivas para la inmigración, cuotas (como en 1965, 1986 y 1996) y el reforzamiento del control fronterizo (como en 1994, 1996 y 2001). Los migrantes mexicanos se convirtieron en los «chivos expiatorios» para los problemas económicos y políticos de Estados Unidos. Al poner en práctica políticas visibles de control de un flujo que había sido manejado negligentemente en el pasado, el gobierno estadounidense logró obtener legitimidad y distraer a la opinión pública de problemas políticos y económicos más profundos (Bustamante, 1983; Andreas, 2000).

A pesar de estas medidas, los flujos migratorios no disminuyeron, sino que los flujos documentados y controlados fueron sustituidos por flujos de indocumentados y sin control (Massey et al., 2002). Como demuestra Meter Andreas, las políticas de Estados Unidos representaban más un símbolo de control político que una política real que reconociera la demanda estructural de fuerza de trabajo (Andreas, 2000). Aun así, tuvieron éxito porque se redujeron las presiones de parte de los patrones y de la opinión pública. Los patrones podían obtener mano de obra todavía más barata y dócil al aprovechar la vulnerabilidad de los migrantes indocumentados y la ausencia de sanciones o controles gubernamentales. Los sindicatos y la opinión pública quedaban satisfechos porque el gobierno parecía controlar el territorio y proteger los intereses y valores de la población. Esta «protección» implicó un incremento en la cantidad de cercas y de patrullas fronterizas y en el número de aprehensiones y deportaciones por parte de la Ins, lo que incrementó los riesgos y los peligros del cruce fronterizo por áreas que quedaban ocultas a la vista del público. Al mantener abierta «la puerta trasera» para ser utilizada en la migración indocumentada, Estados Unidos satisfizo sus necesidades económicas y políticas.

Aun cuando los efectos negativos y las consecuencias no deseadas de estas políticas (para ambos países) fueron examinadas y documentadas exhaustivamente desde que se iniciaron, el gobierno de Estados Unidos conservó e incluso incrementó los controles fronterizos y las políticas restrictivas: tendencia que persiste en la actualidad. Los mitos diseminados, por los medios de comunicación y líderes políticos, acerca del crecimiento de la comunidad mexicana-estadounidense y de la inseguridad y el temor vinculados 
con los cruces de indocumentados, tuvieron como consecuencia un círculo vicioso de presiones para lograr más control de la inmigración. Esto explica la política migratoria «disuasiva» del presidente William Clinton (1992-2000), que consistía en una serie de operaciones de reforzamiento de la frontera y de leyes restrictivas que incrementaron los costos de cruzar la frontera y redujeron los beneficios sociales para los migrantes. ${ }^{5}$ Los costos de estas políticas los asumieron los migrantes y el gobierno mexicano, porque tuvieron como consecuencia mayor maltrato y violencia en contra de los migrantes. Esto incrementó la presión interna en contra de las autoridades mexicanas que, evidentemente, eran incapaces de (o carecían de voluntad para) dominar la situación. Mientras tanto, la legitimidad y el dominio del gobierno de Estados Unidos se consolidaron porque era capaz de «controlar» un problema que se representaba como si fuese una amenaza para la población.

No obstante que el gobierno mexicano estaba consciente de los problemas y costos que significaba una migración continuada y creciente hacia Estados Unidos, y también de las críticas e inestabilidad internas que podían desencadenarse por la larga ausencia de una política migratoria explícita y de programas de desarrollo alternativo para las áreas rurales y las comunidades de origen de los migrantes, sus acciones fueron débiles. Cuando se compara con las políticas de Estados Unidos, la respuesta del gobierno mexicano a estas presiones ejemplifica las capacidades asimétricas de los países para lidiar con los costos de la migración y también su desarrollo histórico, político y cultural particulares.

Desde la década de los cuarenta, el gobierno mexicano había considerado a la migración como una «válvula de seguridad» para los problemas económicos y políticos, hasta que se pudieran desarrollar las condiciones para la distribución equitativa del ingreso y para la justicia social que prometiera la Revolución. A pesar de que el crecimiento económico se sostuvo hasta fines de los sesenta, los beneficios del milagro mexicano no alcanzaron a la población rural, en la que el gobierno revolucionario había fundamentado sus reclamos y su legitimidad. Bajo estas circunstancias, la migración resultó ir más allá de una simple solución temporal y se convirtió, en cambio,

\footnotetext{
${ }^{5}$ La aprobación del Acta de Reforma de la Inmigración Ilegal y de Responsabilidad de la Inmigración de 1996 (IIRIRA) autorizaba financiamiento para diferentes operaciones de control fronterizo y la reducción en los beneficios de la seguridad social, la educación y la asistencia pública para los extranjeros con documentos y sin documentos, al igual que las restricciones al apoyo de la inmigración familiar legal. Esta ley estaba acompañada del Acta de Reconciliación de la Responsabilidad Personal y de Oportunidades de Empleo (PRWORA) de 1996, que estableció límites más estrictos para el acceso de los migrantes documentados e indocumentados a los beneficios federales, estatales y locales. La primera operación para el control fronterizo fue la de «bloqueo» (Blockade) —que más tarde se denominó «Conserva la línea» (Hold the Line) - en el cruce entre El Paso, Texas y Ciudad Juárez, Chihuahua (septiembre 1993), que se extendió en enero de 1997 a Nuevo México. Luego se estableció la «Operación Guardián» (Gatekeeper) en el área entre San Diego, California y Tijuana, Baja California Norte (octubre 1994), que se expandió en octubre de 1996 para cubrir 66 millas. La operación «Salvaguarda» (Safeguard) se puso en práctica entre Nogales, Arizona y Nogales, Sonora (1995), que se expandió en 1999 hacia Douglas y Naco. La última de estas operaciones, «Río Grande», cubrió el área del sureste tejano (agosto 1997). Todas estas operaciones incluían el establecimiento de cercas de acero, iluminación, torres de vigilancia, cámaras de video, sistemas de supervisión de infrarrojos de alta potencia y un aumento en el número de agentes de la patrulla fronteriza (véase Andreas, 2002; Cornelius, 2001).
} 
en una estrategia permanente para reducir las presiones políticas por parte de la ciudadanía. Además, constituyó un factor de importancia para el desarrollo económico, porque aseguró el flujo de divisas extranjeras por medio de las remesas.

Aun cuando los mexicanos se enfrentaron a maltratos y serios riesgos en Estados Unidos durante y después del Programa Bracero, los esfuerzos del gobierno mexicano por protegerlos estuvieron acotados. Parte de las limitaciones eran consecuencia de su vulnerabilidad al negociar con Estados Unidos. No obstante, también es cierto que México prefirió garantizar la permanencia de estos flujos y evitar las tensiones con Estados Unidos que pudieran llevar a repatriaciones masivas y a políticas que impidieran la emigración. Esto no justifica ni explica el hecho de que los gobiernos mexicanos no lograran proponer programas sostenibles de desarrollo en las áreas rurales o en las instituciones nacionales para proteger los derechos de los migrantes. ${ }^{6}$ Aunque México estaba perdiendo una parte importante de su fuerza de trabajo, lo que implicaba cambios sociales y económicos en las comunidades rurales y generaba una mayor dependencia respecto a las remesas, las políticas dirigidas a manejar estas circunstancias no fueron suficientes. Para 1974, tras muchos intentos fallidos por negociar otro programa bilateral de fuerza de trabajo migrante con Estados Unidos, México se había decidido por una vertiente de «no política» hacia la migración (García y Griego, 1988). Ello propició que el tema se conservara con un bajo perfil en la política exterior y en las agendas nacionales, con el objeto de preservar un cómodo statu quo para el gobierno a pesar de los costos para la población.

Tras la caída del modelo económico de sustitución de importaciones, a fines de los años setenta, que llevó a la crisis de la deuda externa en 1982 y obligó a más mexicanos a buscar oportunidades de empleo en Estados Unidos, las soluciones nacionales que se habían ofrecido a los migrantes carecían evidentemente de eficacia. Incluso en esos momentos, la permanencia de la retórica nacionalista y revolucionaria se utilizaría todavía para legitimar los programas de gobierno, que tácitamente asumían a la migración como un «mal necesario» para garantizar la estabilidad económica y política. En el discurso del gobierno, la independencia respecto a Estados Unidos y la defensa de la soberanía nacional se acentuaban de manera notable. Paradójicamente, México comenzó al mismo tiempo un proceso de liberalización económica en los años ochenta que se basaba en el apoyo y la guía de Estados Unidos. Estos cambios constituyeron los antecedentes del proceso que desembocó en el TLCAN. A lo largo de este proceso, el discurso nacionalista comenzó a transformarse para justificar la integración económica con Estados Unidos, pero todavía se limitaron las acciones internas e internacionales del gobierno en áreas como la migración y la energía (Mabire, 1994). Los temas migratorios permanecieron en la periferia, mientras que los costos y las consecuencias negativas de las políticas puestas en práctica se siguieron acumulando silenciosamente en el corazón del sistema mexicano.

Las condiciones y circunstancias nacionales, descritas aquí de manera general, revelan las connotaciones políticas que conlleva el tema migratorio y el hecho de que estén profundamente integradas en las respuestas de los gobiernos. En Estados Unidos, la influencia de los grupos de presión, como los patrones o los sindicatos, y la

${ }^{6}$ Los proyectos, como el establecimiento de maquiladoras (plantas de ensamblado) en la frontera desde 1965, resultaron ineficaces para reducir la migración hacia Estados Unidos. 
existencia de reacciones nativistas o antiinmigrantes, a las crisis económicas o políticas, proporcionan importantes factores explicativos para los resultados de las políticas migratorias. Las limitaciones de México para promover cambios en el statu quo se encuentran en su vulnerabilidad en cualquier negociación de un acuerdo migratorio con Estados Unidos y su interés por mantener las buenas relaciones y la cooperación económica. Además, la necesidad de mantener abierta esta «válvula de seguridad» y evitar las presiones internas han determinado también su pasividad al tratarse de la migración. La asimetría de poder constituyó el contexto en el que se enmarcaron y desarrollaron estas políticas.

El TLCAN reflejó tanto las restricciones internas como las internacionales para lograr una definición común y una política compartida en torno a la migración. El tratado incluyó cambios intrínsecos en la relación bilateral, lo que significó confrontar, de manera novedosa, los desafíos planteados por la migración. El grado al cual estos cambios pueden llevar a la cooperación bilateral formal se examina a continuación.

\section{TRAS EL TLCAN: ¿QUÉ HA CAMBIADO Y QUÉ PUEDE CAMBIAR?}

Como explica Francisco Alba, la iniciativa misma del TLCAN afecta la manera en que se tratan los temas migratorios (Alba, 1993). En una atmósfera favorable para la cooperación y el diálogo, el intercambio de información entre gobiernos, instituciones académicas y comisiones bilaterales se ha extendido gradualmente hasta incluir nuevos actores, como las ONG y las empresas. Iniciativas como la de Border Liaison Mechanisms, la cooperación entre los gobiernos local y estatal en los asuntos regionales, los programas compartidos de entrenamiento para las patrullas fronterizas y planes comunes para las seguridad en las fronteras son ejemplos de la extensión de la cooperación hacia áreas no incluidas en el TLCAN.

Tras el TLCAN, la percepción recíproca de ambos países se ha alterado. Durante las negociaciones del TLCAN, México diseñó nuevas estrategias de cabildeo que contribuyeron a construir nuevas relaciones y redes en Estados Unidos a través del Congreso, los partidos políticos, los medios de comunicación, los sindicatos y otros grupos de presión. México se dio cuenta, también, de la influencia de los líderes y políticos latinos y de la importancia electoral de la comunidad mexicana-estadounidense, tanto en Estados Unidos como en México. ${ }^{7}$ La importancia y la influencia de la población

\footnotetext{
${ }^{7}$ La importancia de la comunidad mexicana-estadounidense se hizo evidente desde los años setenta, pero el interés del gobierno por fortalecer los vínculos con su población no se enfatizó sino hasta principios de los noventa. A través de programas como el dirigido a las Comunidades Mexicanas en el Extranjero (PCME), el Programa Paisano y la creación del Grupo Beta, el gobierno mexicano realizó esfuerzos mayores para organizar a la comunidad mexicana, proteger los derechos de los migrantes y ejercer un mejor control de los cruces fronterizos. La cifra de las representaciones consulares y sus funciones también se incrementó (en la actualidad hay más de 45 representaciones diplomáticas mexicanas en Estados Unidos). Uno de los mayores cambios en la posición del gobierno hacia su población en Estados Unidos quedó demostrado cuando las presiones políticas en contra de los migrantes se manifestaron a través de iniciativas legales como la propuesta 187 en California, en 1994. El gobierno mexicano respondió con la aprobación de una reforma constitucional que
} 
latina para Estados Unidos se evidencia en años recientes debido al hecho de que es ahora la minoría étnica más numerosa (13\% de la población). En los ámbitos gubernamental y social existe un creciente interés por establecer vínculos más estrechos con los latinos y por integrarlos en sus programas, comunidades o asociaciones.

La relevancia de la comunidad mexicana-estadounidense y de la población migrante se hizo sentir en 2001 durante las diversas reuniones entre los presidentes Vicente Fox (2000-2006) y George W. Bush (2000-2004) y su compromiso por una negociación amplia de los temas migratorios. Ambos países habían decidido negociar la propuesta de México a favor de un acuerdo migratorio global, que incluyera la regularización de los migrantes indocumentados que actualmente se encuentran en Estados Unidos, con un incremento en el número de visas permanentes concedidas anualmente a los mexicanos, el establecimiento de programas de trabajadores huéspedes, la creación de nuevas estrategias de seguridad en la frontera y la promoción del desarrollo económico en México, en especial, en aquellas regiones desde las que parten la mayoría de los migrantes (Leiken, 2001).

La estabilidad económica mexicana, después del gobierno del presidente Ernesto Zedillo (1994-2000) y la transición democrática que condujo a la elección del presidente Fox, ofreció al nuevo gobierno la posibilidad de abandonar los restos del discurso nacionalista pasivo y cambiarlo hacia un nuevo activismo, en el que las mejoras al TLCAN y las relaciones con Estados Unidos representaran un factor de peso. La consolidación de un acuerdo migratorio habría de ser un aspecto fundamental de esta nueva política exterior y de la «relación especial» entre Estados Unidos y México. El régimen de Bush parecía seguir esta línea al interesarse en lograr legitimidad gracias al éxito en un tema de política exterior y a través de asegurar para sí el voto latino. ${ }^{8}$

Después de los ataques del 11 de septiembre de 2001, cambiaron las prioridades del gobierno de Estados Unidos y se redujeron las posibilidades de un acuerdo, al menos a corto plazo. No obstante, ya no podía ignorarse más la importancia del tema y su inclusión en la agenda bilateral. Ello se muestra en la discusión del tema migratorio efectuada en reuniones posteriores entre los presidentes y otros actores políticos, al igual que en la actual cooperación en el área de la seguridad fronteriza, las tarjetas de identificación de migrantes (matrículas consulares) y las facilidades para los flujos de remesas. A pesar del estancamiento de las negociaciones para el acuerdo propuesto en 2001, la necesidad de

permite la doble nacionalidad (ello no incluye la doble ciudadanía, lo que significa que los mexicanos que tienen un país de residencia diferente no pueden votar en las elecciones mexicanas). Esta reforma permitió a los ciudadanos mexicanos en Estados Unidos utilizar su voto para ejercer sus derechos y proteger sus intereses y tener un mayor impacto como electorado.

${ }^{8}$ Muchos comentarios editoriales y artículos periodísticos publicados en Estados Unidos interpretaron el interés de Bush en los temas latinos como una estrategia para obtener votos para el Partido Republicano al distanciarlo de su posición tradicional opuesta a la inmigración (por ejemplo, Robert Leiden, «Border Colleagues: On Migration, Bush and Fox Belong on the Same Side», The Washington Post, 2 de septiembre de 2001). Antes de los ataques del 11 de septiembre se veía al acuerdo migratorio como una posibilidad de que Bush lograra una fácil victoria política que pudiera reforzar su debatida legitimidad tras las elecciones de 2000 y, de paso, el débil apoyo político a su partido. Un análisis de estos temas se discutió, por parte de varios académicos, en la reunión «The Political Role of Mexican Americans and the 2001 and 2002 Elections», The New School, Internacional Center for Migration, Ethnicity and Citizenship (ICMEC) Nueva York, 6 de diciembre de 2002. 
un manejo bilateral de la migración se ha convertido en una realidad. La posibilidad de ese desarrollo depende de la voluntad de ambos países y de su capacidad para resolver los temas internos urgentes y para avanzar hacia una integración más plena. Esto no significa, necesariamente, que se incluya un acuerdo de libre mercado laboral en el TLCAN, sino que se logre, en cambio, un manejo conjunto de la situación común.

\section{LOS DESAFÍOS ACTUALES}

Hoy en día, tanto en México como en Estados Unidos, existe más conciencia acerca de las causas y consecuencias de la migración, esa conciencia trasciende las fronteras nacionales. Las consecuencias negativas de que no exista una definición compartida y una puesta en práctica coordinada de la política de migración ya no pueden ser soslayadas. Una razón importante para ello la constituye la magnitud de la población implicada, además de los costos humanos y las violaciones a los derechos humanos que ello ha significado. La otra razón es que la integración de las economías y el incremento en los flujos de bienes, al igual que las comunicaciones transnacionales entre instituciones, empresas, sociedades y gobiernos, conllevan flujos de personas. Para que estos flujos de personas se den de manera segura, controlada y óptima, y para que se logren las metas establecidas en el TLCAN, ambos gobiernos deben acordar y negociar los términos en los que han de responder a estos desafíos.

Una de las más importantes transformaciones ha de ocurrir en la información transmitida por ambos gobiernos a sus ciudadanos. La necesidad de presentar la situación de manera desmitificada y objetiva es una condición para que la gente se involucre y acepte las realidades de la migración. A través de ello será posible resolver temas relacionados con la contratación de mano de obra migrante, las violaciones a los derechos humanos, los controles fronterizos y la asimilación de los migrantes en mejores términos - más susceptibles de manejo-. Para Estados Unidos, esto requeriría el reconocimiento del aspecto de la demanda en el problema y la necesidad de aumentar las cuotas de las visas a la vez que crear programas adecuados de trabajo temporal. Ello implicaría, también, una nueva estrategia para reducir las consecuencias negativas de la vigilancia fronteriza, como se muestra en las más de 2,355 muertes en la frontera desde que comenzaron las operaciones de reforzamiento en 1993 (Cornelius, Reforma, 6 de junio de 2003), el incremento en el contrabando, la corrupción y la violencia en la frontera, además de la prolongada presencia de trabajadores indocumentados. Si Estados Unidos cambiara sus actuales políticas, seguramente surgirían presiones por parte de los patrones, los grupos antiinmigrantes y la opinión pública influida por el discurso político y los medios de comunicación que exageran los costos de la inmigración. Aun así, la voluntad política de parte del poder Ejecutivo y el cabildeo del presidente en el Congreso han mostrado en el pasado su capacidad para reducir estas presiones. ${ }^{9}$

${ }^{9}$ Todd Eisenstadt (2000) muestra cómo fue posible lograr el consenso necesario para la aprobación del TLCAN a pesar de la oposición de diversos grupos y un Congreso dividido. Para lograrlo, Clinton prometió la asignación de recursos y otros beneficios a los congresistas que apoyaran la iniciativa. La voluntad política del presidente fue también un factor clave en 1995, cuando Clinton decidió conceder un préstamo para ayudar a México a resolver su crisis financiera a pesar de la oposición del Congreso. 
Con el objeto de generar las condiciones para que se dé ese proceso, México todavía tiene que mostrar su capacidad para manejar y controlar a su población migrante en las fronteras del norte y del sur, además de continuar desarrollando un marco institucional en esta área. Para México, un acuerdo migratorio formal implicaría aceptar la realidad de sus vínculos con Estados Unidos, los beneficios que traerá consigo la cooperación posterior y los obstáculos que implica mantener el discurso nacionalista a este respecto. No obstante, el presidente Fox y su ex secretario de Relaciones Exteriores, Jorge G. Castañeda (2000-2002), avanzaron de forma notable en esta dirección al hacer un cambio bastante explícito y radical en los objetivos y el discurso de la política exterior. ${ }^{10}$ Para el gobierno mexicano también es importante mostrar a la población, que vive fuera de su territorio, el interés y la prioridad que le da a los temas relacionados con ellos, ya que su influencia política y económica, en ambos, países continúa incrementándose. La creación del Instituto para los Mexicanos en el Extranjero (IME), en abril de 2003, fue un importante avance en esta área.

Incluso cuando en el corto plazo es insuficiente la solución que se plantea para la migración en las negociaciones del TLCAN, sería poco realista pensar que el acuerdo bilateral propuesto en 2001 será la solución final. Independientemente de las pérdidas o los logros en el ámbito de la política exterior, México debe enfrentar los desafíos internos para reducir los costos que se derivan de las políticas del pasado y preparar las condiciones para alcanzar un acuerdo con Estados Unidos que responda a sus propios intereses. Si el gobierno mexicano pone todo su esfuerzo en las negociaciones con Estados Unidos, corre el riesgo de achacar el fracaso de su estrategia al hecho de que éste último no esté dispuesto a negociar. Ello significaría retrasar, todavía más, las soluciones integrales.

En el ámbito interno, México requiere todavía una definición explícita y clara de las estrategias efectivas que habrán de servir para vigilar la seguridad en sus fronteras en el norte y en el sur, reformar las instituciones que tengan que ver con la migración y desarrollar y crear alternativas para el desarrollo en las comunidades de origen. Tales acciones pueden mejorar su posición negociadora en caso del diálogo bilateral y conducirán también a soluciones más amplias para este problema. En lo internacional, el gobierno debe continuar reforzando sus relaciones con grupos en Estados Unidos y en todos los niveles del cabildeo. La información y los recursos disponibles, desde la firma del TLCAN, han sido un instrumento de importancia que desarrollan estrategias exitosas para tratar con el gobierno de Estados Unidos y con otros actores influyentes. En el futuro, como reconoció el ex secretario Castañeda, el sentido de la oportunidad de las estrategias de México será un factor determinante en su éxito (Castañeda, 2001).

Es innegable que Estados Unidos puede seguir confiando en su poder y en su capacidad para controlar los costos de las consecuencias políticas, logrando legitimidad a través de procesos restrictivos, a menos que los costos de hacerlo resulten demasiado altos. Ello obedece a las presiones que se deriven de los grupos nacionales a favor de un acuerdo migratorio y los que se oponen a los cabildeos de los patrones, deseosos de mantener bajos los costos de la mano de obra al contratar inmigrantes

${ }^{10}$ Aunque todavía hay sensibilidades políticas en México que podrían limitar la efectividad de una estrategia basada en una relación más estrecha con Estados Unidos, esto se asocia más con ciertas materias políticas, económicas y comerciales que con los temas migratorios. 
ilegales. También dependerá de la habilidad del gobierno mexicano para convocar a los grupos que favorecen la inmigración en Estados Unidos, de poder atraer el apoyo de otros sectores enfatizando que este país también requiere de México para alcanzar sus metas en el TLCAN, y así lograr los beneficios garantizados por el hecho de que su vecino del sur acceda al crecimiento económico.

Ya no se cuestiona que la migración es un factor permanente y estructural en las relaciones entre México y Estados Unidos, debido a las características de ambos países. Como en el caso del TLCAN, siempre habrá ganadores y perdedores en ambas naciones como resultado de la migración (Rico, 1992). La pregunta ahora es si los gobiernos tienen todavía el poder y la capacidad para controlar quiénes serán los ganadores y los perdedores a través de políticas unilaterales. ¿Puede el manejo bilateral de la migración entre México y Estados Unidos reducir los costos y aportar beneficios para ambos países? A medida que se siga desarrollando el TLCAN y la integración regional, los gobiernos y las sociedades de México y Estados Unidos tendrán que hacer frente a estos desafíos nacionales y bilaterales y decidir en dónde trazar los límites para la cooperación.

\section{REFERENCIAS}

AlbA, Francisco (1993), «El acuerdo comercial: ¿un paso hacia la convergencia sobre la cuestión migratoria?», en Gustavo Vega (ed.), México-Estados Unidos-Canadá, 1991-1992, El Colegio de México, México.

(2000), «Diálogo e incomprensión: el tema migratorio a cuatro años de vigencia del TLC», en Bernardo Mabire (ed.), México-Estados Unidos-Canadá, 1997-1998, El Colegio de México, México.

Andreas, Peter (2000), Border Games: Policing the U.S.-Mexico Divide, Cornell University Press, Ithaca.

Bustamante, Jorge A. (1983), «Mexican Migration: The Political Dynamic of Perceptions», en Clark Reynolds y Carlos Tello (eds.), U.S.-Mexico Relations: Economic and Social Aspects, Stanford University Press, Stanford.

Castañeda, Jorge (2001), «Los ejes de la política exterior de México», en Nexos, núm. 288, México.

Cornelius, Wayne (2000), «Acogida ambivalente: economía, cultura y etnia en las políticas de inmigración de los Estados Unidos», en Foro Internacional, núm. 16. (2001), «The Efficacy and 〈Unintended〉Consequences of U.S. Immigration Control Policy, 1993-2000», en Population and Development Review, núm. 27. (2003), «Las lecciones equivocadas», en Reforma, 9 de junio de 2003.

Corwin, Arthur F. (comp.) (1978), Immigrants-and Immigrants; Perspectives on Mexican Labor Migration to the U.S., Greenwood Press, Westport, Connecticut.

EIsenstadt, Todd A. (2000), «El Auge del Cabildeo Mexicano en Washington: más lejos del cielo y más cerca de Estados Unidos», en Bernardo Mabire (ed.), México-Estados Unidos-Canadá, 1997-1998, El Colegio de México, México.

García y Griego, Manuel y Mónica Vera Campos (1988), México y Estados Unidos frente a la migración de los indocumentados, unAm/Porrúa, México.

KeOHAne, Robert O. (1990), «El concepto de interdependencia y el análisis de las relaciones asimétricas», en Blanca Torres (ed.), Interdependencia ¿un enfoque 
útil para el análisis de las relaciones México-Estados Unidos?, El Colegio de México, México.

KRASNER, Stephen (1990), «Interdependencia simple y obstáculos para la cooperación entre México y Estados Unidos», en Blanca Torres (ed.), Interdependencia ¿un enfoque útil para el análisis de las relaciones México-Estados Unidos?, El Colegio de México, México.

Leiken, Robert (2001), Enchilada Lite: A Post-9/11 Mexican Migration Agreement, Center for Immigration Studies Backgrounder (CIS), Washington, D.C.

MABIRE, Bernardo (1994), «El fantasma de la antigua ideología y su resistencia al cambio de la política exterior en el sexenio de Carlos Salinas de Gortari», en Foro Internacional, núm. 34.

Massey, Douglas S., Jorge Durand y Nolan J. Malone (2002), Beyond Smoke and Mirrors: Mexican Immigration in an Era of Economic Integration, Russell Sage Foundation, Nueva York.

Rico, Carlos (1992), «Migration and U.S.-Mexico Relations, 1966-1986», en Christopher Mitchell (ed.), Western Hemisphere Immigration and u.s Foreign Policy, Pennsylvania State University Press, Pennsylvania. 